\title{
Correspondence
}

\section{On Passivity and Passification of Stochastic Fuzzy Systems With Delays: The Discrete-Time Case}

Jinling Liang, Zidong Wang, and Xiaohui Liu

\begin{abstract}
Takagi-Sugeno (T-S) fuzzy models, which are usually represented by a set of linear submodels, can be used to describe or approximate any complex nonlinear systems by fuzzily blending these subsystems, and so, significant research efforts have been devoted to the analysis of such models. This paper is concerned with the passivity and passification problems of the stochastic discrete-time T-S fuzzy systems with delay. We first propose the definition of passivity in the sense of expectation. Then, by utilizing the Lyapunov functional method, the stochastic analysis combined with the matrix inequality techniques, a sufficient condition in terms of linear matrix inequalities is presented, ensuring the passivity performance of the T-S fuzzy models. Finally, based on this criterion, state feedback controller is designed, and several criteria are obtained to make the closed-loop system passive in the sense of expectation. The results acquired in this paper are delay dependent in the sense that they depend on not only the lower bound but also the upper bound of the time-varying delay. Numerical examples are also provided to demonstrate the effectiveness and feasibility of our criteria.
\end{abstract}

Index Terms-Discrete-time fuzzy system, linear matrix inequality (LMI), Lyapunov functional, passivity, stochastic disturbance, timevarying delay.

\section{INTRODUCTION}

The last decade has seen a wealth of research on passivity and passification problems in the area of systems and control. The reason is mainly twofold: 1) passivity is an expected system behavior, since the storage function induced by passivity is closely related to system energy and therefore serves as a natural candidate for Lyapunov functions, and 2) stability and stabilization problems can be solved once the passivity property is assured. The passivity theory was first proposed in the circuit analysis [1] and has then been applied in many areas such as stability, signal processing, complexity, fuzzy control, chaos control, and synchronization [2], [5], [28], [30]. Recently, it has been recognized that the time delays exist naturally in various engineering and biological systems which constitute a source of instability. Therefore, the passivity and passification problems have been considered in [6], [22] for continuous linear/neutral time-delay systems with or without parameter uncertainties. Note that the passivity analysis has also been conducted in [23] for neural networks, in [7] under the topic of positive realness, and in [8], [9] for networked control systems.

In the past 30 years, the fuzzy-logic theory has been proven to be effective in dealing with the analysis and synthesis problems of

Manuscript received March 4, 2008; revised May 5, 2008 and August 27, 2008. This work was supported in part by the Royal Society Sino-British Fellowship Trust Award of the U.K., by the National Natural Science Foundation of China under Grant 60804028, by the Specialized Research Fund for the Doctoral Program of Higher Education for New Teachers in China under Grant 200802861044, and by the Teaching and Research Fund for Excellent Young Teachers at Southeast University of China. This paper was recommended by Associate Editor H. Gao.

J. Liang is with the Department of Mathematics, Southeast University, Nanjing 210096, China (e-mail: jinlliang@ gmail.com).

$\mathrm{Z}$. Wang and $\mathrm{X}$. Liu are with the Department of Information Systems and Computing, Brunel University, UB8 3PH Uxbridge, U.K. (e-mail: zidong.wang@brunel.ac.uk).

Digital Object Identifier 10.1109/TSMCB.2009.2033142 nonlinear systems. In particular, the Takagi-Sugeno (T-S) fuzzy model has used a set of IF-THEN rules that are formed from linguistic variables and values by quantifying the meaning of the linguistic values using membership functions. As a result, the conventional linear system theory can be applied to the analysis and synthesis of the class of nonlinear systems, and numerous nonlinear analysis problems have been studied based on this T-S fuzzy model. For example, the robust stability analysis and synthesis problems have been addressed in [3] for T-S fuzzy models, and the time delay has been further introduced in [4]. Recently, there has been a rich body of results on the stability problems for time-delay fuzzy T-S systems by using various delaydependent or delay-independent approaches (see, e.g., [14] and [19] for a survey). In addition, since discrete-time systems have come to play a more important role than their continuous-time counterparts in today's digital world, the stability analysis results on discrete-time time-delay fuzzy-model-based control systems have recently drawn an increasing research interest (see, e.g., [10], [13], [17], [33]).

Given the conceptual importance of passivity theory and the practical convenience of T-S fuzzy model, it seems quite natural to generalize the passivity theory to T-S fuzzy models with or without time delays. For instance, the passivity for continuous-time T-S fuzzy systems with constant delays has been considered in [18]. On the other hand, since Itô-type stochastic systems are suitable in modeling many practical systems in engineering, biology, and economy, the robust stability, stabilization, and control, as well as filtering problems for stochastic systems, have been intensively investigated, and many results have been reported in the literature (see, e.g., [25]-[27]). In particular, stochastic fuzzy T-S systems with or without time delays have recently received much attention [15], [16], [24], [32]. For example, in [16] and [24], both parameter uncertainties and stochastic disturbances have been studied for the T-S model, and the exponential mean-square stability has been discussed in [24]. In [15], the slidingmode-control problem for nonlinear stochastic time-delay systems has been dealt with by means of fuzzy approach.

It should be pointed out that most of aforementioned literatures have been concerned with the continuous-time systems only. To date, there have been very few results on the passivity and passification problems of discrete-time T-S fuzzy systems with or without time delays, not to mention the case when stochastic disturbances are taken into account. It is, therefore, the purpose of this paper to pave a way for investigating the passivity problem of discrete stochastic T-S fuzzy systems.

In this paper, we aim to deal with the passivity and passification problems of the stochastic discrete-time T-S fuzzy systems with timevarying delay. First, we define the so-called stochastic passivity (i.e., in the sense of expectation) for discrete-time stochastic systems. We then derive several sufficient conditions in order to make sure that the nominal and controlled T-S fuzzy systems are globally passive in the sense of expectation, where the Lyapunov functional method and stochastic analysis tools are utilized. The obtained conditions are expressed in terms of linear matrix inequalities (LMIs) that can be readily solved by using MATLAB LMI toolbox.

Notations: Throughout this paper, $I_{m}$ is the $m$-dimensional identity matrix. $P>0$ means that matrix $P$ is real, symmetric, and positive definite. Let $\mathbb{E}\{\cdot\}$ be the mathematical expectation operator with respect to the given probability measure $\mathcal{P}$, and $(\Omega, \mathcal{F}, \mathcal{P})$ be a complete probability space with a natural filtration $\left\{\mathcal{F}_{t}\right\}_{t \geq 0}$. For integers $\alpha, \beta$ with $\alpha<\beta, \mathbb{N}[\alpha, \beta]$ denotes the discrete interval given by $\mathbb{N}[\alpha, \beta]=\{\alpha, \alpha+1, \ldots, \beta-1, \beta\}$. Sometimes, when no confusion 
would arise, the dimensions of a function or a matrix will be omitted for convenience.

\section{Problem Formulation And Preliminaries}

In this section, we consider a discrete-time T-S fuzzy system with stochastic disturbances and time-varying delay with the $i$ th rule formulated in the following form:

Plant Rule $i$ :

IF $\theta_{1}(k)$ is $\eta_{i 1}$ and $\ldots \theta_{p}(k)$ is $\eta_{i p}$, THEN

$$
\left\{\begin{array}{c}
x(k+1)=A_{i} x(k)+C_{i} x(k-\tau(k))+G_{i} J(k) \\
\quad+\sigma_{i}(k, x(k), x(k-\tau(k))) \omega_{i}(k) \\
y(k)=B_{i} x(k)+D_{i} x(k-\tau(k))+W_{i} J(k) \\
x(k)=\varphi(k), \quad k \in \mathbb{N}\left[-\tau_{2}, 0\right]
\end{array}\right.
$$

where $i=1,2, \ldots, r ; k=1,2, \ldots$, and $r$ is the number of IF-THEN rules; $\theta(k)=\left\{\theta_{1}(k), \theta_{2}(k), \ldots, \theta_{p}(k)\right\}$ is the premise variable set and $\eta_{i j}(j=1,2, \ldots, p)$ is the fuzzy set; $x(k) \in \mathbb{R}^{n}$ is the state vector and $y(k) \in \mathbb{R}^{m}$ is the measurement output vector; $\varphi(\cdot)$ represents the initial function and $J(k) \in \mathbb{R}^{m}$ is a deterministic exogenous input; and $\omega_{i}(k)$ is a scalar Wiener process (Brownian motion) defined on the complete probability space $(\Omega, \mathcal{F}, \mathcal{P})$ with

$$
\begin{aligned}
\mathbb{E}\left\{\omega_{i}(k)\right\} & =0 \\
\mathbb{E}\left\{\omega_{i}^{2}(k)\right\} & =1 \\
\mathbb{E}\left\{\omega_{i}(l) \omega_{i}(k)\right\} & =0, \quad(l \neq k) .
\end{aligned}
$$

Moreover, the noise processes $\omega_{1}(k), \omega_{2}(k), \ldots, \omega_{r}(k)$ are independent. It is assumed that the premise variables do not depend on the noise-input variables $\omega_{i}(k)$ explicitly [15], [16], [24], [32]. The diffusion coefficient vector $\sigma_{i}(\cdot, \cdot, \cdot): \mathbb{R} \times \mathbb{R}^{n} \times \mathbb{R}^{n} \rightarrow \mathbb{R}^{n}$ satisfies the Lipschitz condition, and there exist matrices $\Sigma_{i 1}$ and $\Sigma_{i 2}$ of appropriate dimensions such that

$$
\sigma_{i}^{\mathrm{T}}(t, u, v) \sigma_{i}(t, u, v) \leq\left\|\Sigma_{i 1} u\right\|^{2}+\left\|\Sigma_{i 2} v\right\|^{2}
$$

for all $(t, u, v) \in \mathbb{R} \times \mathbb{R}^{n} \times \mathbb{R}^{n} . A_{i}, C_{i}, G_{i}, B_{i}, D_{i}$, and $W_{i}$ are system matrices with compatible dimensions, and $\tau(k)$ is the timevarying delay satisfying

$$
\tau_{1}<\tau(k)<\tau_{2}
$$

where $\tau_{1}$ and $\tau_{2}$ are known positive integers.

Let

$$
\mu_{i}(\theta(k))=\prod_{j=1}^{p} \eta_{i j}\left(\theta_{j}(k)\right) \quad h_{i}(\theta(k))=\frac{\mu_{i}(\theta(k))}{\sum_{j=1}^{r} \mu_{j}(\theta(k))}
$$

where $\eta_{i j}\left(\theta_{j}(k)\right)$ is the grade of membership of $\theta_{j}(k)$ in $\eta_{i j}$. It is always assumed that $\mu_{i}(\theta(k)) \geq 0 \quad(i=1,2, \ldots, r)$ and $\sum_{j=1}^{r} \mu_{j}(\theta(k))>0$ for all $k=1,2, \ldots$ Obviously, one has that for $i=1,2, \ldots, r$ and $k=1,2, \ldots$

$$
h_{i}(\theta(k)) \geq 0 \quad \sum_{i=1}^{r} h_{i}(\theta(k))=1 .
$$

Then, the defuzzied output of the T-S fuzzy system (1) can be represented as (5), shown at the bottom of the page.

In the literature, different definitions of passivity have been used. Taking into account the stochastic nature of the T-S fuzzy systems considered in this paper, we define the following notion of passivity in the sense of expectation, which is an extension of the concept of passivity proposed in [21], [31] to the stochastic systems.

Definition 1: The fuzzy system (1) is called globally passive in the sense of expectation if there exists a scalar $\gamma \geq 0$ such that

$$
2 \sum_{k=0}^{T} \mathbb{E}\left\{y^{\mathrm{T}}(k) J(k)\right\} \geq-\gamma \sum_{k=0}^{T} \mathbb{E}\left\{J^{\mathrm{T}}(k) J(k)\right\}
$$

for all integers $T \geq 0$ and the solution of (1) with $\varphi \equiv 0$.

Remark 1: The Itô-type stochastic noise $\omega_{i}(k)$ appears in the state equation. It would be interesting to look at the case when the stochastic noise also enters into the output equation, which is more complicated and deserves further investigation.

Before starting the main results, we need to introduce the following lemma which will be used in the next section.

Lemma 1 [13]: For any real matrices $X_{i}, Y_{i}$, and $P>0$ with compatible dimensions, then

$$
2 \sum_{i=1}^{r} \sum_{j=1}^{r} \gamma_{i} \gamma_{j} X_{i}^{\mathrm{T}} P Y_{j} \leq \sum_{i=1}^{r} \gamma_{i}\left(X_{i}^{\mathrm{T}} P X_{i}+Y_{i}^{\mathrm{T}} P Y_{i}\right)
$$

where $\gamma_{i}(i=1,2, \ldots, r)$ are nonnegative scalars with $\sum_{i=1}^{r} \gamma_{i}=1$.

\section{PASSIVITy AnALYSis}

In this section, based on the Lyapunov functional method, the stochastic analysis combined with the matrix-inequality techniques, we will derive a delay-dependent passivity criterion which will play an important role in the passification problem of the stochastic discrete-time T-S fuzzy system (1). For simplicity, let $\sigma_{i}(k)$ denote $\sigma_{i}(k, x(k), x(k-\tau(k)))$.

Theorem 1: The system (1) is globally passive in the sense of expectation if there exist matrices $P_{i}>0, Q>0, R_{1}>0, R_{2}>0$, $Z>0$; matrices $M, N$; and scalars $\gamma \geq 0, \lambda_{i}>0$ such that the following LMIs hold for all $i, l=1,2, \ldots, r$ :

$$
P_{i}+\tau_{2} Z<\lambda_{i} I
$$

$$
\left[\begin{array}{ccccc}
\Xi_{i l} & \left(\tau_{2}-\tau_{1}\right) M & \tau_{2} N & \bar{A}_{i}^{\mathrm{T}} P_{l} & \tau_{2} \tilde{A}_{i}^{\mathrm{T}} Z \\
* & -\left(\tau_{2}-\tau_{1}\right) Z & 0 & 0 & 0 \\
* & * & -\tau_{2} Z & 0 & 0 \\
* & * & * & -P_{l} & 0 \\
* & * & * & * & -\tau_{2} Z
\end{array}\right]<0
$$

where $\Xi_{i l}=\Phi_{i l}+M T_{1}+T_{1}^{\mathrm{T}} M^{\mathrm{T}}+N T_{2}+T_{2}^{\mathrm{T}} N^{\mathrm{T}}$

$$
\Phi_{i l}=\left[\begin{array}{ccccc}
\tilde{\Xi}_{i l} & 0 & 0 & 0 & -B_{i}^{\mathrm{T}} \\
* & -R_{1} & 0 & 0 & 0 \\
* & * & \lambda_{l} \Sigma_{i 2}^{\mathrm{T}} \Sigma_{i 2}-Q & 0 & -D_{i}^{\mathrm{T}} \\
* & * & * & -R_{2} & 0 \\
* & * & * & * & \hat{\Xi}_{i}
\end{array}\right]
$$

$$
\left\{\begin{array}{l}
x(k+1)=\sum_{i=1}^{r} h_{i}(\theta(k))\left[A_{i} x(k)+C_{i} x(k-\tau(k))+G_{i} J(k)+\sigma_{i}(k, x(k), x(k-\tau(k))) \omega_{i}(k)\right] \\
y(k)=\sum_{i=1}^{r} h_{i}(\theta(k))\left[B_{i} x(k)+D_{i} x(k-\tau(k))+W_{i} J(k)\right]
\end{array}\right.
$$


and $\tilde{\Xi}_{i l}=\left(\tau_{2}-\tau_{1}+1\right) Q+R_{1}+R_{2}-P_{i}+\lambda_{l} \Sigma_{i 1}^{\mathrm{T}} \Sigma_{i 1}, \hat{\Xi}_{i}=-\gamma I-$ $\left(W_{i}+W_{i}^{\mathrm{T}}\right) ; \bar{A}_{i}=\left[\begin{array}{lllll}A_{i} & 0 & C_{i} & 0 & G_{i}\end{array}\right], \tilde{A}_{i}=\left[\begin{array}{llll}\left(A_{i}-I\right) & 0 & C_{i}\end{array}\right.$ $\left.0 \quad G_{i}\right], T_{1}=\left[\begin{array}{lllll}0 & 0 & I & -I & 0\end{array}\right]$, and $T_{2}=\left[\begin{array}{lllll}I & 0 & -I & 0 & 0\end{array}\right]$.

Proof: Denoting $\quad \xi^{\mathrm{T}}(k)=\left(\begin{array}{lll}x^{\mathrm{T}}(k) & x^{\mathrm{T}}\left(k-\tau_{1}\right) \quad x^{\mathrm{T}}(k-\end{array}\right.$ $\left.\tau(k)) \quad x^{\mathrm{T}}\left(k-\tau_{2}\right) \quad J^{\mathrm{T}}(k)\right)$ and by the well-known Schur Lemma, we know that LMI (7) is equivalent to the following inequality:

$\Phi_{i l}+M T_{1}+T_{1}^{\mathrm{T}} M^{\mathrm{T}}+N T_{2}+\left(\tau_{2}-\tau_{1}\right) M Z^{-1} M^{\mathrm{T}}$

$$
+T_{2}^{\mathrm{T}} N^{\mathrm{T}}+\tau_{2} N Z^{-1} N^{\mathrm{T}}+\bar{A}_{i}^{\mathrm{T}} P_{l} \bar{A}_{i}+\tau_{2} \tilde{A}_{i}^{\mathrm{T}} Z \tilde{A}_{i}<0 .
$$

Choose a Lyapunov functional $V(k, x(k))$ as

$$
V(k, x(k))=\sum_{i=1}^{4} V_{i}(k, x(k))
$$

where

$$
\begin{aligned}
V_{1}(k, x(k)) & =x^{\mathrm{T}}(k)\left(\sum_{i=1}^{r} h_{i}(\theta(k)) P_{i}\right) x(k) \\
V_{2}(k, x(k)) & =\sum_{i=k-\tau(k)}^{k-1} x^{\mathrm{T}}(i) Q x(i)+\sum_{j=k-\tau_{2}+1}^{k-\tau_{1}} \sum_{i=j}^{k-1} x^{\mathrm{T}}(i) Q x(i) \\
V_{3}(k, x(k)) & =\sum_{i=k-\tau_{1}}^{k-1} x^{\mathrm{T}}(i) R_{1} x(i)+\sum_{i=k-\tau_{2}}^{k-1} x^{\mathrm{T}}(i) R_{2} x(i) \\
V_{4}(k, x(k)) & =\sum_{j=-\tau_{2}}^{-1} \sum_{i=k+j}^{k-1} \eta^{\mathrm{T}}(i) Z \eta(i)
\end{aligned}
$$

with $\eta(i)=x(i+1)-x(i)$.

Calculating the difference of $V_{i}(k, x(k))(i=1,2,3,4)$ along the trajectories of (5), taking the mathematical expectation and noticing that

$$
\begin{aligned}
x(k+1) & =\sum_{i=1}^{r} h_{i}(\theta(k))\left[\bar{A}_{i} \xi(k)+\sigma_{i}(k) \omega_{i}(k)\right] \\
\eta(k) & =\sum_{i=1}^{r} h_{i}(\theta(k))\left[\tilde{A}_{i} \xi(k)+\sigma_{i}(k) \omega_{i}(k)\right]
\end{aligned}
$$

we have

$$
\begin{aligned}
\mathbb{E}\left\{\Delta V_{1}(k, x(k))\right\}=\mathbb{E}\{ & \sum_{l=1}^{r} h_{l}^{+} \sum_{i=1}^{r} \sum_{j=1}^{r} h_{i}(\theta(k)) h_{j}(\theta(k)) \\
& \times\left[\xi^{\mathrm{T}}(k) \bar{A}_{i}^{\mathrm{T}} P_{l} \bar{A}_{j} \xi(k)+\sigma_{i}^{\mathrm{T}}(k) P_{l} \sigma_{i}(k)\right] \\
& \left.-\sum_{i=1}^{r} h_{i}(\theta(k)) x^{\mathrm{T}}(k) P_{i} x(k)\right\} \\
\leq \mathbb{E}\{ & \sum_{l=1}^{r} h_{l}^{+} \sum_{i=1}^{r} h_{i}(\theta(k)) \\
& \times\left[\xi^{\mathrm{T}}(k) \bar{A}_{i}^{\mathrm{T}} P_{l} \bar{A}_{i} \xi(k)-x^{\mathrm{T}}(k) P_{i} x(k)\right. \\
& \left.\left.+\sigma_{i}^{\mathrm{T}}(k) P_{l} \sigma_{i}(k)\right]\right\}
\end{aligned}
$$

where $h_{l}^{+}=h_{l}(\theta(k+1))$. Note that, to obtain (12), Lemma 1 and the independent property of stochastic processes $\omega_{1}(k), \omega_{2}(k), \ldots, \omega_{r}(k)$ have been utilized.
Similarly, we can obtain that

$$
\begin{aligned}
& \mathbb{E}\left\{\Delta V_{2}(k, x(k))\right\} \\
& =\mathbb{E}\left\{x^{\mathrm{T}}(k) Q x(k)-x^{\mathrm{T}}(k-\tau(k)) Q x(k-\tau(k))\right. \\
& +\left(\sum_{i=k+1-\tau(k+1)}^{k-\tau_{1}}+\sum_{i=k-\tau_{1}+1}^{k-1}-\sum_{i=k-\tau(k)+1}^{k-1}\right) x^{\mathrm{T}}(i) Q x(i) \\
& \left.+\sum_{j=1-\tau_{2}}^{-\tau_{1}}\left(\sum_{i=k+1-j}^{k}-\sum_{i=k+j}^{k-1}\right) x^{\mathrm{T}}(i) Q x(i)\right\} \\
& \leq \mathbb{E}\left\{\left(\tau_{2}-\tau_{1}+1\right) x^{\mathrm{T}}(k) Q x(k)\right. \\
& \left.-x^{\mathrm{T}}(k-\tau(k)) Q x(k-\tau(k))\right\} \\
& \mathbb{E}\left\{\Delta V_{3}(k, x(k))\right\} \\
& =\mathbb{E}\left\{x^{\mathrm{T}}(k)\left(R_{1}+R_{2}\right) x(k)-x^{\mathrm{T}}\left(k-\tau_{1}\right) R_{1} x\left(k-\tau_{1}\right)\right. \\
& \left.-x^{\mathrm{T}}\left(k-\tau_{2}\right) R_{2} x\left(k-\tau_{2}\right)\right\} \\
& \mathbb{E}\left\{\Delta V_{4}(k, x(k))\right\} \\
& =\mathbb{E}\left\{\tau_{2} \eta^{\mathrm{T}}(k) Z \eta(k)-\sum_{i=k-\tau_{2}}^{k-1} \eta^{\mathrm{T}}(i) Z \eta(i)\right\} \\
& \leq \mathbb{E}\left\{\tau_{2} \sum_{i=1}^{r} h_{i}(\theta(k))\left[\xi^{\mathrm{T}}(k) \tilde{A}_{i}^{\mathrm{T}} Z \tilde{A}_{i} \xi(k)+\sigma_{i}^{\mathrm{T}}(k) Z \sigma_{i}(k)\right]\right. \\
& \left.-\sum_{i=k-\tau_{2}}^{k-\tau(k)-1} \eta^{\mathrm{T}}(i) Z \eta(i)-\sum_{i=k-\tau(k)}^{k-1} \eta^{\mathrm{T}}(i) Z \eta(i)\right\} .
\end{aligned}
$$

On the other hand, for any matrices $M$ and $N$ with appropriate dimensions, the following equalities hold:

$$
\begin{aligned}
& 2 \xi^{\mathrm{T}}(k) M\left(x(k-\tau(k))-x\left(k-\tau_{2}\right)-\sum_{i=k-\tau_{2}}^{k-\tau(k)-1} \eta(i)\right)=0 \\
& 2 \xi^{\mathrm{T}}(k) N\left(x(k)-x(k-\tau(k))-\sum_{i=k-\tau(k)}^{k-1} \eta(i)\right)=0 .
\end{aligned}
$$

Combing (12)-(17) yields

$$
\begin{aligned}
& \mathbb{E}\{\Delta V(k, x(k))\} \\
& \leq \mathbb{E}\left\{\sum_{l=1}^{r} h_{l}^{+} \sum_{i=1}^{r} h_{i}(\theta(k))\right. \\
& \quad \times\left[\xi^{\mathrm{T}}(k)\left(\bar{A}_{i}^{\mathrm{T}} P_{l} \bar{A}_{i}+\tau_{2} \tilde{A}_{i}^{\mathrm{T}} Z \tilde{A}_{i}\right) \xi(k)\right. \\
& \quad+2 \xi^{\mathrm{T}}(k) M T_{1} \xi(k)+2 \xi^{\mathrm{T}}(k) N T_{2} \xi(k) \\
& \quad+\sigma_{i}^{\mathrm{T}}(k)\left(P_{l}+\tau_{2} Z\right) \sigma_{i}(k)-x^{\mathrm{T}}\left(k-\tau_{1}\right) R_{1} x\left(k-\tau_{1}\right) \\
& \quad+x^{\mathrm{T}}(k)\left(\left(\tau_{2}-\tau_{1}+1\right) Q-P_{i}+R_{1}+R_{2}\right) x(k) \\
& \quad-x^{\mathrm{T}}(k-\tau(k)) Q x(k-\tau(k)) \\
& \left.\left.\quad-x^{\mathrm{T}}\left(k-\tau_{2}\right) R_{2} x\left(k-\tau_{2}\right)\right]\right\} \\
& -\sum_{i=k-\tau_{2}} \mathbb{E}\left\{\eta^{\mathrm{T}}(i) Z \eta(i)+2 \xi^{\mathrm{T}}(k) M \eta(i)\right\} \\
& -\sum_{i=k-\tau(k)}^{k-1} \mathbb{E}\left\{\eta^{\mathrm{T}}(i) Z \eta(i)+2 \xi^{\mathrm{T}}(k) N \eta(i)\right\} .
\end{aligned}
$$


From the conditions (3) and (6), it follows:

$$
\begin{aligned}
\mathbb{E} & \left\{\sigma_{i}^{\mathrm{T}}(k)\left(P_{l}+\tau_{2} Z\right) \sigma_{i}(k)\right\} \\
& \leq \lambda_{l} \mathbb{E}\left\{\sigma_{i}^{\mathrm{T}}(k) \sigma_{i}(k)\right\} \\
& \leq \lambda_{l} \mathbb{E}\left\{x^{\mathrm{T}}(k) \Sigma_{i 1}^{\mathrm{T}} \Sigma_{i 1} x(k)+x^{\mathrm{T}}(k-\tau(k)) \Sigma_{i 2}^{\mathrm{T}} \Sigma_{i 2} x(k-\tau(k))\right\}
\end{aligned}
$$

Substituting (19) into (18) and noticing (8), one has

$$
\begin{aligned}
\mathbb{E}\{\Delta & \left.V(k, x(k))-2 y^{\mathrm{T}}(k) J(k)-\gamma J^{\mathrm{T}}(k) J(k)\right\} \\
\leq \mathbb{E} & \left\{\sum_{l=1}^{r} h_{l}^{+} \sum_{i=1}^{r} h_{i}(\theta(k)) \xi^{\mathrm{T}}(k)\right. \\
& \times\left[\Phi_{i l}+M T_{1}+T_{1}^{\mathrm{T}} M^{\mathrm{T}}+\tau_{2} \tilde{A}_{i}^{\mathrm{T}} Z \tilde{A}_{i}\right. \\
+ & \left.\left.\bar{A}_{i}^{\mathrm{T}} P_{l} \bar{A}_{i}+N T_{2}+T_{2}^{\mathrm{T}} N^{\mathrm{T}}\right] \xi(k)\right\} \\
+ & \sum_{i=k-\tau_{2}} \mathbb{E}\left\{\xi^{\mathrm{T}}(k) M Z^{-1} M^{\mathrm{T}} \xi(k)\right\} \\
+ & \sum_{i=k-\tau(k)}^{k-1} \mathbb{E}_{k}\left\{\xi^{\mathrm{T}}(k) N Z^{-1} N^{\mathrm{T}} \xi(k)\right\} \\
\leq & \left\{\sum_{l=1}^{r} h_{l}^{+} \sum_{i=1}^{r} h_{i}(\theta(k)) \xi^{\mathrm{T}}(k)\right. \\
& \times\left[\Phi_{i l}+\bar{A}_{i}^{\mathrm{T}} P_{l} \bar{A}_{i}+M T_{1}+\tau_{2} \tilde{A}_{i}^{\mathrm{T}} Z \tilde{A}_{i}+T_{1}^{\mathrm{T}} M^{\mathrm{T}}\right. \\
+ & N T_{2}+T_{2}^{\mathrm{T}} N^{\mathrm{T}}+\left(\tau_{2}-\tau_{1}\right) M Z^{-1} M^{\mathrm{T}} \\
&
\end{aligned}
$$

and therefore we have

$$
\begin{aligned}
& 2 \sum_{k=0}^{T} \mathbb{E}\left\{y^{\mathrm{T}}(k) J(k)\right\} \\
& \quad \geq \sum_{k=0}^{T} \mathbb{E}\{\Delta V(k, x(k))\}-\gamma \sum_{k=0}^{T} \mathbb{E}\left\{J^{\mathrm{T}}(k) J(k)\right\} \\
& \quad \geq-\mathbb{E}\{V(0, x(0))\}-\gamma \sum_{k=0}^{T} \mathbb{E}\left\{J^{\mathrm{T}}(k) J(k)\right\} \\
& \quad=-\gamma \sum_{k=0}^{T} \mathbb{E}\left\{J^{\mathrm{T}}(k) J(k)\right\}
\end{aligned}
$$

for all integers $T \geq 0$. From Definition 1, (20) implies that the stochastic fuzzy system (1) is globally passive in the sense of expectation, and the proof is then completed.

Example 1: In order to illustrate Theorem 1, we consider the stochastic discrete-time fuzzy system (1) with $r=2$, where $r$ is the number of IF-THEN rules. The time-varying delay $\tau(k)$ is assumed to have $\tau_{1}=2$ and $\tau_{2}=5$. Other parameters are given as follows:

$$
\begin{array}{ll}
A_{1}=\left[\begin{array}{cc}
-0.21 & 0.12 \\
0 & -0.19
\end{array}\right] \quad A_{2}=\left[\begin{array}{cc}
-0.23 & 0 \\
0.1 & -0.15
\end{array}\right] \\
C_{1}=\left[\begin{array}{cc}
0.12 & -0.12 \\
-0.1 & 0.1
\end{array}\right] \quad C_{2}=\left[\begin{array}{cc}
-0.13 & 0.1 \\
-0.1 & 0.12
\end{array}\right]
\end{array}
$$

$$
\begin{aligned}
G_{1} & =\left[\begin{array}{cc}
0.1 & 0.2 \\
0.15 & -0.14
\end{array}\right] \quad G_{2}=\left[\begin{array}{cc}
-0.2 & -0.1 \\
0.18 & -0.23
\end{array}\right] \\
B_{1} & =\left[\begin{array}{cc}
-0.4 & 0.1 \\
0.12 & -0.31
\end{array}\right] \quad B_{2}=\left[\begin{array}{cc}
-0.25 & 0.16 \\
0.05 & -0.36
\end{array}\right] \\
D_{1} & =\left[\begin{array}{cc}
-0.35 & 0.13 \\
0.16 & -0.29
\end{array}\right] \quad D_{2}=\left[\begin{array}{cc}
0.1 & -0.11 \\
0.17 & -0.4
\end{array}\right] \\
W_{1} & =\left[\begin{array}{cc}
0.21 & -0.1 \\
0.1 & 0.31
\end{array}\right] \quad W_{2}=\left[\begin{array}{cc}
-0.42 & 0 \\
0.11 & 0.32
\end{array}\right] .
\end{aligned}
$$

The noise diffusion coefficient vectors $\sigma_{i}(\cdot, \cdot, \cdot)(i=1,2)$ satisfy the condition (3) with

$$
\begin{aligned}
& \Sigma_{11}=\Sigma_{21}=\left[\begin{array}{cc}
0.1 & 0.16 \\
-0.1 & 0.21
\end{array}\right] \\
& \Sigma_{12}=\Sigma_{22}=\left[\begin{array}{cc}
0.2 & 0.13 \\
-0.15 & -0.3
\end{array}\right] .
\end{aligned}
$$

By using the MATLAB LMI Toolbox, LMIs (6) and (7) can be solved with the feasible solutions. For simplicity, only some of the solutions are given as follows:

$$
P_{1}=\left[\begin{array}{cc}
969.4 & 18.4 \\
18.4 & 1117.9
\end{array}\right] \quad \lambda_{1}=1190.8 .
$$

According to Theorem 1, the discrete-time fuzzy model (1) with parameters above is globally passive in the sense of expectation.

\section{PASSIFICATION}

Now, we are ready to consider the passification problem, i.e., the problem of designing a state feedback controller that makes the closedloop fuzzy system passive. Consider the following stochastic discretetime T-S fuzzy model with control input:

\section{Plant Rule $i$ :}

$$
\begin{aligned}
& \text { IF } \theta_{1}(k) \text { is } \eta_{i 1} \text { and } \ldots \theta_{p}(k) \text { is } \eta_{i p}, \text { THEN } \\
& \qquad \begin{array}{r}
x(k+1)=A_{i} x(k)+C_{i} x(k-\tau(k))+G_{i} J(k) \\
+\sigma_{i}(k) \omega_{i}(k)+S_{i} u(k) \\
y(k)=B_{i} x(k)+D_{i} x(k-\tau(k))+W_{i} J(k)
\end{array}
\end{aligned}
$$

where $k=1,2, \ldots ; i=1,2, \ldots, r ; S_{i}$ is a constant matrix with appropriate dimensions, and $u(k) \in \mathbb{R}^{l}$ is the control input.

In this paper, the state feedback controller is taken to be as follows:

$$
u(k)=\sum_{j=1}^{r} h_{j}(\theta(k)) K_{j} x(k) .
$$

Then, the closed-loop fuzzy system can be represented as

$$
\left\{\begin{aligned}
x(k+1)=\sum_{i=1}^{r} & \sum_{j=1}^{r} h_{i}(\theta(k)) h_{j}(\theta(k)) \\
& \times\left[G_{i} J(k)+C_{i} x(k-\tau(k))\right. \\
& \left.+\left(A_{i}+S_{i} K_{j}\right) x(k)+\sigma_{i}(k) \omega_{i}(k)\right]
\end{aligned}\right.
$$

Theorem 2: The feedback closed-loop fuzzy system (23) is globally passive in the sense of expectation if there exist matrices $P_{i}>0, X_{i}>$ $0, Q>0, R_{1}>0, R_{2}>0, Z>0, Y>0$; matrices $M, N, K_{i}$; and 
scalars $\gamma \geq 0, \lambda_{i}>0$ such that the following matrix inequalities hold for all $i, j, l=1,2, \ldots, r$ :

$$
\begin{aligned}
& P_{i}+\tau_{2} Z<\lambda_{i} I \\
& P_{i} X_{i}=I \quad Z Y=I \\
& {\left[\begin{array}{ccccc}
\Xi_{i l} & \left(\tau_{2}-\tau_{1}\right) M & \tau_{2} N & \bar{A}_{i j}^{\mathrm{T}} & \tau_{2} \tilde{A}_{i j}^{\mathrm{T}} \\
* & -\left(\tau_{2}-\tau_{1}\right) Z & 0 & 0 & 0 \\
* & * & -\tau_{2} Z & 0 & 0 \\
* & * & * & -X_{l} & 0 \\
* & * & * & * & -\tau_{2} Y
\end{array}\right]<0}
\end{aligned}
$$

where $\quad \bar{A}_{i j}=\left[\begin{array}{lllll}\left(A_{i}+S_{i} K_{j}\right) & 0 & C_{i} & 0 & G_{i}\end{array}\right], \quad \tilde{A}_{i j}=\left[\left(A_{i}+\right.\right.$ $\left.\left.S_{i} K_{j}-I\right) \quad 0 \quad C_{i} \quad 0 \quad G_{i}\right] ; \Xi_{i l}$ is defined as that in Theorem 1.

Proof: From (23) and the definition of $\eta(k)$, one has

$$
\begin{aligned}
x(k+1) & =\sum_{i=1}^{r} \sum_{j=1}^{r} h_{i}(\theta(k)) h_{j}(\theta(k))\left[\bar{A}_{i j} \xi(k)+\sigma_{i}(k) \omega_{i}(k)\right] \\
\eta(k) & =\sum_{i=1}^{r} \sum_{j=1}^{r} h_{i}(\theta(k)) h_{j}(\theta(k))\left[\tilde{A}_{i j} \xi(k)+\sigma_{i}(k) \omega_{i}(k)\right] .
\end{aligned}
$$

Choose the same Lyapunov functional $V(k, x(k))$ as in Theorem 1 . Calculating the difference of $V_{1}(k, x(k))$ along the solutions of (23) and taking the mathematical expectation, we have

$$
\begin{aligned}
& \mathbb{E}\{\Delta\left.V_{1}(k, x(k))\right\} \\
&=\mathbb{E}\left\{\sum_{l=1}^{r} h_{l}^{+} \sum_{i=1}^{r} \sum_{j=1}^{r} \sum_{p=1}^{r} \sum_{q=1}^{r}\right. \\
& \times h_{i}(\theta(k)) h_{j}(\theta(k)) h_{p}(\theta(k)) h_{q}(\theta(k)) \\
&\left.\times\left[\xi^{\mathrm{T}}(k) \bar{A}_{i j}^{\mathrm{T}} P_{l} \bar{A}_{p q} \xi(k)+\sigma_{i}^{\mathrm{T}}(k) P_{l} \sigma_{i}(k)-x^{\mathrm{T}}(k) P_{i} x(k)\right]\right\} \\
& \leq \mathbb{E}\left\{\sum_{l=1}^{r} h_{l}^{+} \sum_{i=1}^{r} \sum_{j=1}^{r} h_{i}(\theta(k)) h_{j}(\theta(k))\right. \\
&\left.\times\left[\xi^{\mathrm{T}}(k) \bar{A}_{i j}^{\mathrm{T}} P_{l} \bar{A}_{i j} \xi(k)+\sigma_{i}^{\mathrm{T}}(k) P_{l} \sigma_{i}(k)-x^{\mathrm{T}}(k) P_{i} x(k)\right]\right\} .
\end{aligned}
$$

Here, to obtain (29), Lemma 1 has been utilized.

Along the similar lines of the proof of Theorem 1, we can get

$$
\begin{aligned}
\mathbb{E}\left\{\Delta V(k, x(k))-2 y^{\mathrm{T}}(k) J(k)-\gamma J^{\mathrm{T}}(k) J(k)\right\} \\
\leq \mathbb{E}\left\{\sum_{l=1}^{r} h_{l}^{+} \sum_{i=1}^{r} \sum_{j=1}^{r} h_{i}(\theta(k)) h_{j}(\theta(k)) \xi^{\mathrm{T}}(k)\right. \\
\quad \times\left[\Phi_{i l}+M T_{1}+\bar{A}_{i j}^{\mathrm{T}} P_{l} \bar{A}_{i j}+\tau_{2} \tilde{A}_{i j}^{\mathrm{T}} Z \tilde{A}_{i j}+T_{1}^{\mathrm{T}} M^{\mathrm{T}}+N T_{2}\right. \\
\left.\left.\quad+T_{2}^{\mathrm{T}} N^{\mathrm{T}}+\left(\tau_{2}-\tau_{1}\right) M Z^{-1} M^{\mathrm{T}}+\tau_{2} N Z^{-1} N^{\mathrm{T}}\right] \xi(k)\right\} .
\end{aligned}
$$

By noting that $P_{l}=X_{l}^{-1}, Z=Y^{-1}$, and from the well-known Schur Lemma, we know that conditions (24)-(26) ensure that $\mathbb{E}\left\{\Delta V(k, x(k))-2 y^{\mathrm{T}}(k) J(k)-\gamma J^{\mathrm{T}}(k) J(k)\right\} \leq 0$. The remaining part of the proof of Theorem 2 is similar to that of Theorem 1 and is therefore omitted.
Remark 2: The conditions acquired in Theorem 2 are not strict LMIs, but they can be computed by resorting to the cone complementarity linearization (CCL) method which could also be found in [10].

An alternative computational procedure for the passification is given in the following corollary.

Corollary 1: The feedback closed-loop fuzzy system (23) is globally passive in the sense of expectation if there exist matrices $\bar{P}>0$, $\bar{Q}>0, \bar{R}_{1}>0, \bar{R}_{2}>0$; matrices $F_{j}$; and scalars $\gamma \geq 0, \bar{\lambda}>0$ such that the following LMIs hold for all $i, j=1,2, \ldots, r$ :

$$
\bar{P}>\bar{\lambda} I
$$

$$
\left[\begin{array}{ccccccc}
\Gamma^{\prime} & 0 & 0 & 0 & -\bar{P} B_{i}^{\mathrm{T}} & \check{\Xi}_{i j} & \aleph_{i 1} \\
* & -\bar{R}_{1} & 0 & 0 & 0 & 0 & 0 \\
* & * & -\bar{Q} & 0 & -\bar{P} D_{i}^{\mathrm{T}} & \bar{P} C_{i}^{\mathrm{T}} & \aleph_{i 2} \\
* & * & * & -\bar{R}_{2} & 0 & 0 & 0 \\
* & * & * & * & \hat{\Xi}_{i} & G_{i}^{\mathrm{T}} & 0 \\
* & * & * & * & * & -\bar{P} & 0 \\
* & * & * & * & * & * & -\bar{\lambda} I
\end{array}\right]<0
$$

where $\Gamma^{\prime}=\left(\tau_{2}-\tau_{1}+1\right) \bar{Q}+\bar{R}_{1}+\bar{R}_{2}-\bar{P}, \check{\Xi}_{i j}=\bar{P} A_{i}^{\mathrm{T}}+F_{j} S_{i}^{\mathrm{T}}$, $\aleph_{i 1}=\left[\bar{P} \Sigma_{i 1}^{\mathrm{T}}, 0\right], \aleph_{i 2}=\left[0, \bar{P} \Sigma_{i 2}^{\mathrm{T}}\right]$, and $\hat{\Xi}_{i}$ is defined in Theorem 1 . Moreover, the feedback controller gain can be constructed as

$$
K_{j}=F_{j}^{\mathrm{T}} \bar{P}^{-1} .
$$

Remark 3: Compared to Theorem 2, the conditions expressed in Corollary 1 are expressed in terms of LMIs which could be more easily computed and checked in practice at the expense of making $P_{l} \equiv P(l=1,2, \ldots, r)$ and losing some free matrices, i.e., $Z=0$, $M=N=0$.

Remark 4: Using the methods given earlier, it is not difficult to deal with the robust passivity and passification problems of uncertain stochastic discrete-time delayed fuzzy systems as follows:

\section{Plant Rule $i$ :}

$$
\begin{aligned}
& \text { IF } \theta_{1}(k) \text { is } \eta_{i 1} \text { and } \ldots \theta_{p}(k) \text { is } \eta_{i p}, \text { THEN } \\
& \qquad \begin{array}{r}
x(k+1)=\left(\begin{array}{r}
\left.A_{i}+\Delta A_{i}\right) x(k)+\left(C_{i}+\Delta C_{i}\right) x(k-\tau(k)) \\
+\left(G_{i}+\Delta G_{i}\right) J(k)+\sigma_{i}(k) \omega_{i}(k)
\end{array}\right. \\
y(k)=\left(\begin{array}{r}
B_{i} \\
\left.+\Delta B_{i}\right) x(k)+\left(D_{i}+\Delta D_{i}\right) x(k-\tau(k)) \\
+\left(W_{i}+\Delta W_{i}\right) J(k), \quad i=1,2, \ldots, r
\end{array}\right.
\end{array}
\end{aligned}
$$

where $k=1,2, \ldots ; \Delta A_{i}, \Delta C_{i}, \Delta G_{i}, \Delta B_{i}, \Delta D_{i}$, and $\Delta W_{i}$ are real matrices representing the norm-bounded parameter uncertainties. To tackle the parameter uncertainties, the techniques in [20] could be borrowed, and the results are omitted here to keep this paper concise.

Example 2: Consider the passification problem of the stochastic discrete-time closed-loop fuzzy system (23) with parameters as in Example 1. Take

$$
S_{1}=\left[\begin{array}{cc}
0.1 & -0.2 \\
-0.1 & 0.11
\end{array}\right] \quad S_{2}=\left[\begin{array}{cc}
-0.13 & 0.11 \\
0.2 & 0.1
\end{array}\right] \text {. }
$$

By using the MATLAB LMI Toolbox, LMIs (30) and (31) can be solved with feasible solutions. According to Corollary 1, we can construct a state feedback controller to make the closed-loop fuzzy system (23) passive with the feedback gain as follows:

$$
\begin{aligned}
K_{1} & =\left[\begin{array}{cc}
-0.1365 & -0.0157 \\
-0.0120 & 0.0412
\end{array}\right] \\
K_{2} & =\left[\begin{array}{cc}
-0.2280 & -0.0714 \\
-0.0228 & 0.2583
\end{array}\right] .
\end{aligned}
$$




\section{CONCLUSION}

In this paper, the passivity and passification problems have been investigated for a general class of stochastic discrete-time fuzzy systems with time-varying delay. To reflect more realistic dynamical behaviors of the system, stochastic disturbances have been considered in the form of Brownian motions. By employing the Lyapunov functional method combined with the matrix-inequality techniques, several sufficient criteria have been acquired to ensure the original T-S fuzzy system and the closed-loop fuzzy model to be globally passive in the sense of expectation. Further research topics include extending the main results of this paper to a class of stochastic or descriptor systems [11], [12].

\section{REFERENCES}

[1] V. Bevelevich, Classical Network Synthesis. New York: Van Nostrand, 1968.

[2] G. Calcev, "Passivity approach to fuzzy control systems," Automatica, vol. 34, no. 3, pp. 339-344, Mar. 1998.

[3] Y. Cao and P. M. Frank, "Robust $H_{\infty}$ disturbance attenuation for a class of uncertain discrete-time fuzzy systems," IEEE Trans. Fuzzy Syst., vol. 8, no. 4, pp. 406-415, Aug. 2000.

[4] Y. Cao and P. M. Frank, "Analysis and synthesis of nonlinear time-delay systems via fuzzy control approach," IEEE Trans. Fuzzy Syst., vol. 8, no. 2, pp. 200-211, Apr. 2000.

[5] L. O. Chua, "Passivity and complexity," IEEE Trans. Circuits Syst. I, Fundam. Theory Appl., vol. 46, no. 1, pp. 71-82, Jan. 1999.

[6] E. Fridman and U. Shaked, "On delay-dependent passivity," IEEE Trans. Autom. Control, vol. 47, no. 4, pp. 664-669, Apr. 2002.

[7] H. Gao, J. Lam, C. Wang, and S. Xu, "Control for stability and positivity: Equivalent conditions and computation," IEEE Trans. Circuits Syst. II, Exp. Briefs, vol. 52, no. 9, pp. 540-544, Sep. 2005.

[8] H. Gao, T. Chen, and J. Lam, "A new delay system approach to networkbased control," Automatica, vol. 44, no. 1, pp. 39-52, Jan. 2008.

[9] H. Gao, T. Chen, and T. Chai, "Passivity and passification for networked control systems," SIAM J. Control Optim., vol. 46, no. 4, pp. 1299-1322, Sep. 2007.

[10] H. Gao, Z. Wang, and C. H. Wang, "Improved $H_{\infty}$ control of discretetime fuzzy systems: A cone complementarity linearization approach," Inf. Sci., vol. 175, no. 1/2, pp. 57-77, Sep. 2005.

[11] Z. Gao, H. Wang, and T. Chai, "A robust fault detection filtering for stochastic distribution systems via descriptor estimator and parametric gain design," IET Control Theory Appl., vol. 1, no. 5, pp. 1286-1293, Sep. 2007.

[12] Z. Gao and S. X. Ding, "State and disturbance estimator for timedelay systems with application to fault estimation and signal compensation," IEEE Trans. Signal Process., vol. 55, no. 12, pp. 5541-5551, Dec. 2007.

[13] X. Guan and C. L. Chen, "Delay-dependent guaranteed cost control for T-S fuzzy systems with time delays," IEEE Trans. Fuzzy Syst., vol. 12, no. 2, pp. 236-249, Apr. 2004.

[14] D. W. C. Ho and J. Sun, "Stability of Takagi-Sugeno fuzzy delay systems with impulse," IEEE Trans. Fuzzy Syst., vol. 15, no. 5, pp. 784-790, Oct. 2007.
[15] D. W. C. Ho and Y. Niu, "Robust fuzzy design for nonlinear uncertain stochastic systems via sliding-mode control," IEEE Trans. Fuzzy Syst., vol. 15, no. 3, pp. 350-358, Jun. 2007.

[16] H. Huang and D. W. C. Ho, "Delay-dependent robust control of uncertain stochastic fuzzy systems with time-varying delay," IET Control Theory Appl., vol. 1, no. 4, pp. 1075-1085, Jul. 2007.

[17] J. Lam and S. S. Zhou, "Dynamic output feedback $H_{\infty}$ control of discrete-time fuzzy systems: A fuzzy-basis-dependent Lyapunov function approach," Int. J. Syst. Sci., vol. 38, no. 1, pp. 25-37, Jan. 2007.

[18] C. Li, H. Zhang, and X. Liao, "Passivity and passification of fuzzy systems with time delays," Comput. Math. Appl., vol. 52, no. 6/7, pp. 1067-1078, Sep. 2006.

[19] C. Lin, Q. G. Wang, T. H. Lee, and Y. He, LMI Approach to Analysis and Control of Takagi-Sugeno Fuzzy Systems With Time Delay, vol. 351, Lecture Notes in Control and Information Sciences. New York: Springer-Verlag, 2007.

[20] Y. Liu, Z. Wang, and X. Liu, "Robust stability of discrete-time stochastic neural networks with time-varying delays," Neurocomputing, vol. 71, no. 4-6, pp. 823-833, Jan. 2008.

[21] R. Lozano, B. Brogliato, O. Egeland, and B. Maschke, Dissipative Systems Analysis and Control: Theory and Applications. London, U.K.: Springer-Verlag, 2000.

[22] S. I. Niculescu and R. Lozano, "On the passivity of linear delay systems," IEEE Trans. Autom. Control, vol. 46, no. 3, pp. 460-464, Mar. 2001.

[23] J. H. Park, "Further results on passivity analysis of delayed cellular neural networks," Chaos Solitons Fractals, vol. 34, no. 5, pp. 1546-1551, Dec. 2007.

[24] Z. Wang, D. W. C. Ho, and X. Liu, "A note on the robust stability of uncertain stochastic fuzzy systems with time-delays," IEEE Trans. Syst., Man, Cybern. A, Syst., Humans, vol. 34, no. 4, pp. 570-576, Jul. 2004.

[25] Z. Wang, Y. Liu, and X. Liu, " $H_{\infty}$ filtering for uncertain stochastic time-delay systems with sector-bounded nonlinearities," Automatica, vol. 44, no. 5, pp. 1268-1277, May 2008.

[26] Z. Wang, F. Yang, D. W. C. Ho, S. Swift, A. Tucker, and X. Liu, "Stochastic dynamic modeling of short gene expression time series data," IEEE Trans. Nanobiosci., vol. 7, no. 1, pp. 44-55, Mar. 2008.

[27] Z. Wang, F. Yang, D. W. C. Ho, and X. Liu, "Robust $H_{\infty}$ control for networked systems with random packet losses," IEEE Trans. Syst., Man, Cybern. B, Cybern., vol. 37, no. 4, pp. 916-924, Aug. 2007.

[28] C. W. Wu, "Synchronization in arrays of coupled nonlinear systems: Passivity, circle criterion, and observer design," IEEE Trans. Circuits Syst. I, Fundam. Theory Appl., vol. 48, no. 10, pp. 1257-1261, Oct. 2001.

[29] H. Wu and K. Y. Cai, " $H_{2}$ guaranteed cost fuzzy control design for discrete-time nonlinear systems with parameter uncertainty," Automatica, vol. 42, no. 7, pp. 1183-1188, Jul. 2006.

[30] L. Xie, M. Y. Fu, and H. Z. Li, "Passivity analysis and passification for uncertain signal processing systems," IEEE Trans. Signal Process., vol. 46, no. 9, pp. 2394-2403, Sep. 1998.

[31] I. Yaesh and U. Shaked, "Stochastic passivity and its application in adaptive control," in Proc. 44th IEEE Conf. Decision Control, 2005, pp. 2871-2876.

[32] H. Zhang, Y. Wang, and D. Liu, "Delay-dependent guaranteed cost control for uncertain stochastic fuzzy systems with multiple time delays," IEEE Trans. Syst., Man, Cybern. B, Cybern., vol. 38, no. 1, pp. 126-140, Feb. 2008.

[33] S. S. Zhou, J. Lam, and A. Xue, " $H_{\infty}$ filtering of discrete-time fuzzy systems via basis-dependent Lyapunov function approach," Fuzzy Sets Syst., vol. 158, no. 2, pp. 180-193, Jan. 2007. 